\title{
Functional repair of a mutant chloride channel using a trans-splicing ribozyme
}

\author{
Christopher S. Rogers, ${ }^{1,2}$ Carlos G. Vanoye, ${ }^{1}$ Bruce A. Sullenger, ${ }^{3}$ \\ and Alfred L. George, Jr. ${ }^{1,2}$ \\ ${ }^{1}$ Division of Genetic Medicine, Department of Medicine, Vanderbilt University, Nashville, Tennessee, USA \\ ${ }^{2}$ Department of Pharmacology, Vanderbilt University, Nashville, Tennessee, USA \\ ${ }^{3}$ Department of Surgery, Duke University, Durham, North Carolina, USA
}

\begin{abstract}
RNA repair has been proposed as a novel gene-based therapeutic strategy. Modified Tetrabymena group I intron ribozymes have been used to mediate trans-splicing of therapeutically relevant RNA transcripts, but the efficiency of the ribozyme-mediated RNA repair process has not been determined precisely and subsequent restoration of protein function has been demonstrated only by indirect means. We engineered a ribozyme that targets the mRNA of a mutant canine skeletal muscle chloride channel (cClC-1) (mutation T268M in ClC-1 causing myotonia congenita) and replaces the mutant-containing $3^{\prime}$ portion by trans-splicing the corresponding 4-kb wild-type sequence. Repair efficiency assessed by quantitative RT-PCR was $1.2 \% \pm 0.1 \%$ in a population of treated cells. However, when chloride channel function was examined in single cells, a wide range of electrophysiological activity was observed, with $18 \%$ of cells exhibiting significant functional restoration and some cells exhibiting complete rescue of the biophysical phenotype. These results indicate that RNA repair can restore wild-type protein activity and reveal considerable cell-to-cell variability in ribozyme-mediated trans-splicing reaction efficiency.
\end{abstract}

J. Clin. Invest. 110:1783-1789 (2002). doi:10.1172/JCI200216481.

\section{Introduction}

Advances in molecular genetics have fostered the development of new therapeutic approaches, such as genebased therapy, for the treatment of inherited disorders. The most widely used strategy involves the introduction of wild-type sequence into cells with a defective gene. This strategy is best suited for the treatment of inherited diseases in which there is complete absence of functional alleles, such as in recessive disorders. However, it is not clear that this approach will succeed in all disorders, especially those with a dominant-negative mechanism of disease pathogenesis.

Strategies using repair of mRNA are alternative approaches that will conceptually work in both dominant and recessive disorders (1). Replacing the defective portion of an mRNA molecule reduces the fraction of mutant transcripts and increases the absolute level of the normal (or repaired) transcript. The advantages of this strategy are that the target mRNA remains under the control of its endogenous promoter, thereby assuring proper regulation of expression, and tissue specificity is achieved by targeting mRNA only in cells expressing the

Received for publication July 23, 2002, and accepted in revised form October 22, 2002.

Address correspondence to: Alfred L. George, Jr., Division of Genetic Medicine, 529 Light Hall, Vanderbilt University, Nashville, Tennessee 37232-0275, USA. Phone: (615) 936-2660; Fax: (615) 936-2661; E-mail: al.george@vanderbilt.edu.

Conflict of interest: The authors have declared that no conflict of interest exists.

Nonstandard abbreviations used: internal guide sequence (IGS); canine skeletal muscle chloride channel (cClC-1); enhanced green fluorescent protein (EGFP); untranslated region (UTR). mutant gene. RNA repair has been accomplished by two similar methods: spliceosome-mediated (2) and ribozyme-mediated trans-splicing (3-6).

The Tetrabymena group I intron ribozyme has been modified to facilitate trans-splicing of its $3^{\prime}$ exon onto separate RNA molecules in a sequence-specific manner $(7,8)$. This trans-splicing reaction is initiated by complementary base pairing of the target RNA to the ribozyme internal guide sequence (IGS). The ribozyme then catalyzes the cleavage of the target RNA immediately downstream of the IGS binding site and the subsequent ligation of its $3^{\prime}$ exon to the remaining target RNA, generating a complete repaired RNA transcript. Ribozyme-mediated transsplicing has been used to repair defective $\beta$-globin (9), myotonic dystrophy protein kinase (10), and p53 transcripts (11) in cultured mammalian cells. To date, the longest trans-spliced $3^{\prime}$ exon has been $1.1 \mathrm{~kb}$ (11), but the ability of the Tetrahymena ribozyme to mediate trans-splicing of larger exons has not been determined. Furthermore, the efficiency of the trans-splicing reaction has not been accurately measured in a cell-based system. Importantly, repaired transcripts must be translated into functional protein, but only one study has demonstrated restored protein function determined indirectly in lysates from a population of cells (11).

To further investigate the feasibility of RNA repair, we designed and tested the ability of a modified Tetrahymena ribozyme to mediate trans-splicing repair of a mutant canine skeletal muscle chloride channel (cClC-1) mRNA transcript that causes the inherited disorder myotonia congenita. We demonstrated that this ribozyme can mediate trans-splicing of a large $(4 \mathrm{~kb}) 3^{\prime}$ exon in cultured cells. Furthermore, we measured trans-splicing reaction 
efficiency in cells and assayed chloride channel function at the single-cell level. Although the overall reaction efficiency determined in a population of cells was low (1.2\%), several cells exhibited partial or complete restoration of normal channel function. These findings demonstrate that RNA repair can fully restore wild-type protein activity in cells containing mutant RNA. However, they also indicate a high degree of cell-to-cell variability in ribozyme-mediated trans-splicing. What accounts for this variability is unclear, but a better understanding of the requirements for such genetic repair in mammalian cells may allow the development of RNA repair strategies that can restore wild-type function to a greater percentage of treated cells.

\section{Methods}

Site accessibility assay. A random-IGS RNA mapping library was constructed using the Tetrabymena group I trans-splicing ribozyme such that the $5^{\prime}$ end of the ribozyme began with 5'-GNNNNN-3' (G represents guanine and N represents equal amounts of the four nucleotides: adenine, guanine, cytidine, or uridine) as previously described (9). The test $3^{\prime}$ exon in this experiment consisted of human ClC-1 (hClC-1) sequence (nucleotides 2,674-2,998). For in vitro transcription of the target RNA, Bsu36I linearized plasmid encoding canine $\mathrm{ClC}-1$ ( $\mathrm{cClC}-1$ ) was used as template. The RNA mapping library and $\mathrm{cClC}-1 \mathrm{RNA}$ were in vitro transcribed using T7 polymerase with the MEGAscript system (Ambion Inc., Austin, Texas, USA). A 30-fold excess of ribozyme library and cClC-1 RNAs were denatured separately at $95^{\circ} \mathrm{C}$ for 1 minute and then pre-equilibrated in reaction buffer (in mM: 50 HEPES, $\mathrm{pH} 7.0,150 \mathrm{NaCl}$, and $5 \mathrm{MgCl}_{2}$ ) at $37^{\circ} \mathrm{C}$ for 2 minutes. The substrates were added to the ribozyme library along with guanosine $(100 \mu \mathrm{M})$ and incubated at $37^{\circ} \mathrm{C}$ for 4 hours. Trans-splicing reaction products were reverse transcribed using AMV-RT (Promega Corp., Madison, Wisconsin, USA) and PCR amplified (Advantage 2 Polymerase; CLONTECH Laboratories Inc., Palo Alto, California, USA) using the following primers: reverse transcription, hClC-1 2855R; forward, cClC-1 -92F; reverse, hClC-1 2772R (primer sequences available on request). PCR products were subcloned into a plasmid vector (PCR2.1-TOPO-TA; Invitrogen Corp., Carlsbad, California, USA) and sequenced.

Ribozyme construction. Active ( $\left.\mathrm{Rz}_{712} \mathrm{AS}\right)$ and inactive $\left(\mathrm{Rz}_{\mathrm{d}} \mathrm{U} 7_{712} \mathrm{AS}\right)$ ribozymes were generated using PCR mutagenesis to yield a product containing the entire Tetrabymena intron (catalytic core) with a $\mathrm{U}_{712}$-targeted ninenucleotide IGS, P10 helix, and 33-nucleotide antisense region on the $5^{\prime}$ end. Plasmids $\mathrm{pT} / \mathrm{S}$ or $\mathrm{pT} / \mathrm{S}_{\mathrm{d}}$ (generous gifts from T. Cech, Howard Hughes Medical Institute, Chevy Chase, Maryland, USA) were used as templates. One portion of the $3^{\prime}$ exon was generated using PCR mutagenesis to introduce 15 silent mutations into the $5^{\prime}$ end of a cClC-1 fragment. Mammalian expression plasmids encoding the active ( $\mathrm{pRcCMV}-\mathrm{RzU}_{712} \mathrm{AS}$ ) and catalytically inactive ( $\mathrm{pRcCMV}-\mathrm{Rz}_{\mathrm{d}} \mathrm{U}_{712} \mathrm{AS}$ ) ribozymes were constructed by ligation of HindIII-ScaI-digested catalytic core, ScaI-AgeI-digested cClC-1 3' exon, and AgeI-HindIIIdigested pRcCMV-cClC- 1 fragments. The final constructs contain a 4,005-base 3 ' exon consisting of cClC- 1 nucleotides 713-4,717. Dual promoter bicistronic plasmids encoding both enhanced green fluorescent protein (EGFP) and either active (pCMS-EGFP-RzU $\mathrm{H}_{712} \mathrm{AS}$ ) or inactive (pCMS-EGFP- $\mathrm{Rz}_{\mathrm{d}} \mathrm{U}_{712} \mathrm{AS}$ ) ribozymes were constructed by subcloning a ribozyme-containing HindIIIXbaI fragment that includes a 3,217-base 3' exon into the multiple cloning site of PCMS-EGFP (CLONTECH Laboratories Inc.). All ribozyme sequences were confirmed by DNA sequence analysis.

In vitro RNA repair. T268M-stable HEK-293 cells (stably expressing T268M) and HEK-293 cells were transfected with $8 \mu \mathrm{g}$ of either pRcCMV-RzU $\mathrm{U}_{712} \mathrm{AS}$ or pRcCMV- $\mathrm{Rz}_{\mathrm{d}} \mathrm{U}_{712} \mathrm{AS}$ using Lipofectamine 2000 (Invitrogen Corp.) in 60-mm dishes. Following trypsinization, total RNA was isolated from cells after 20 hours with the RNAqueous-4PCR reagent (Ambion Inc.). As a control reaction, $\mathrm{pRcCMV}-\mathrm{RzU}_{712} \mathrm{AS}$-transfected HEK-293 cells and nontransfected T268M-stable cells were mixed immediately prior to RNA isolation. RNA was reverse transcribed with a cClC-1 $3^{\prime}$ exon-specific primer (cClC-1-1556R) using AMV-RT (Promega Corp.). cDNA was amplified for 35 cycles with an allelespecific primer set that amplifies only trans-spliced products (cClC-1-279F and SJ6-R or ST-R) using Advantage 2 Polymerase (CLONTECH Laboratories Inc.). Trans-spliced products were subcloned into plasmid vectors and sequenced using M13F and M13R primers.

Quantitative RT-PCR. PCR primers and TaqMan probes were designed using Primer Express version 1.5 (Applied Biosystems, Foster City, California, USA). The fluorescent reporter dye at the $5^{\prime}$ end of the probe is VIC and the quencher dye at the $3^{\prime}$ end is TAMRA. Amplification and detection were performed with an ABI Prism 7700 Sequence Detection System (Applied Biosystems). Total RNA (800 ng) isolated from pCMS-EGFP-RzU 712 AS- or pCMS-EGFP- $\mathrm{Rz}_{\mathrm{d}} \mathrm{U}_{712} \mathrm{AS}$-transfected T268M-stable cells was reverse transcribed and amplified in a $50-\mu 1$ reaction using TaqMan One-Step RT-PCR Master Mix reagents (Applied Biosystems) with the following cycling parameters: 30 minutes at $48^{\circ} \mathrm{C}, 10$ minutes at $95^{\circ} \mathrm{C}$, and 40 cycles of 15 seconds at $95^{\circ} \mathrm{C}$ and 1 minute at $59^{\circ} \mathrm{C}$. The final concentrations of primers and probe were $900 \mathrm{nM}$ and $250 \mathrm{nM}$, respectively. Control reactions without template were always included to ensure that reaction components were free of DNA contamination. The threshold cycle number was determined using Sequence Detection System software (Applied Biosystems) with the baseline set at ten SDs above background level measured during the first 3-15 cycles. Standard curves were generated by plotting the threshold cycle number values against the log of the amount of input plasmid DNA representing either trans-spliced or nonspliced products, or ribozyme or T268M RNA. Relative amounts of unknown RNA samples were inferred from the standard curve formulas: trans-spliced products, $y=-1.4165 \ln (x)$ +31.735 ; nonspliced products, $y=-1.4692 \ln (x)+31.755$; 
ribozyme, $y=-1.3365 \ln (x)+36.735$; and T268M RNA, $y=-1.4126 \ln (x)+38.185$. The proportion of trans-splice junction sequence to total target sequence was calculated as follows: percent RNA repair $=[$ trans-spliced products/(trans-spliced products + nonspliced products, corrected for transfection efficiency)] $\times 100$. The ratio of ribozyme to T268M RNA was calculated as follows: Ratio $=$ ribozyme $/$ T268M RNA, corrected for transfection efficiency. The dual promoter bicistronic plasmids, pCMS-EGFP-RzU ${ }_{712} A S$ and pCMS-EGFP- $z_{\mathrm{d}} \mathrm{U}_{712} \mathrm{AS}$, encode EGFP as a separate transcript, allowing transfection efficiency to be determined as the percentage of green cells measured using a hemocytometer under ultraviolet epifluorescence.

Electrophysiological studies. Whole-cell currents in cells expressing cClC-1 (wild-type, T268M mutant, and T268M mutant with either pCMS-EGFP-RzU ${ }_{712} \mathrm{AS}$ or pCMS-EGFP- $\left.\mathrm{Rz}_{\mathrm{d}} \mathrm{U}_{712} \mathrm{AS}\right)$ were recorded with an Axopatch 200A (Axon Instruments Inc., Foster City, California, USA) in the whole-cell configuration of the patch clamp technique (12). In the ribozyme transfection studies, only EGFP-positive cells were analyzed. Data acquisition and analysis were performed using pCLAMP 7.0 (Axon Instruments Inc.). Additional data analyses were performed using SigmaPlot 2000 (SPSS Science, Chicago, Illinois, USA) and Origin 6.0 (Microcal Software Inc., Northampton, Massachusetts, USA) software packages. Whole-cell currents were filtered at 2 $\mathrm{kHz}$ and acquired at $5 \mathrm{kHz}$. The bath solution contained (in $\mathrm{mM}$ ): $140 \mathrm{NaCl}, 4.0 \mathrm{KCl}, 2.0 \mathrm{CaCl}_{2}, 1.0 \mathrm{MgCl}_{2}, 5$ HEPES, and 10 glucose, $\mathrm{pH} 7.4, \sim 280 \mathrm{mosmol} / \mathrm{kg}$. The pipette solution contained (in $\mathrm{mM}$ ): $130 \mathrm{NaCl}, 2.0$ $\mathrm{MgCl}_{2}, 5 \mathrm{HEPES}$, and 5 EGTA, pH 7.4, 270 mosmol $/ \mathrm{kg}$. The pipette solution was diluted $7-9 \%$ with distilled water to prevent activation of swelling-activated $\mathrm{Cl}^{-}$currents. Patch pipettes were pulled from thick-wall borosilicate glass (World Precision Instruments, Sarasota, Florida, USA) with a multistage P-87 Flaming-Brown micropipette puller (Sutter Instrument Co., San Rafael, California, USA) and fire polished with a microforge (MF-830; Narishige Co., Tokyo, Japan). Pipette resistance was approximately $4 \mathrm{M} \Omega$ with $\mathrm{NaCl}$ in the pipette and bath solutions. Series resistance was always compensated at least $85 \%$. As a reference electrode, an agar bridge with composition similar to the bath solution was used. All experiments were carried out at room temperature.

For initial recordings, currents were elicited with a series of 250-ms test pulses from a holding potential of $0 \mathrm{mV}$ through the range of -160 to $+120 \mathrm{mV}$ in $20-\mathrm{mV}$ steps. The voltage dependence of activation for $\mathrm{CClC}-1$ channels was obtained as previously described (13). Briefly, instantaneous currents were measured at -120 $\mathrm{mV}$ after 1,400 -ms prepulses $(-160$ to $+120 \mathrm{mV}, 20 \mathrm{mV}$ steps, 15 seconds at $0 \mathrm{mV}$ between sweeps) and divided by the largest current value. The normalized data were then plotted versus the prepulse potentials. This plot yields the relative open probability $\left(P_{\mathrm{o}}\right)$ of cClC- 1 channels at the end of the prepulse potential. Using Origin 6.0 software, the data obtained in this manner from each cell were fitted to the Boltzmann equation: $P_{\mathrm{o}}=\left(P_{\mathrm{o}, \min }-P_{\mathrm{o}, \max }\right) /\left\{1+\exp \left[\left(V-V_{1 / 2}\right) \times k_{\mathrm{V}}\right]\right\}+P_{\mathrm{o}, \max }$, where $P_{\mathrm{o}}$ is relative open probability, $P_{\mathrm{o}, \min }$ is minimum $P_{\mathrm{o}}, P_{\mathrm{o}, \max }$ is maximum $P_{\mathrm{o}}, k_{\mathrm{V}}$ is slope factor, and $V_{1 / 2}$ is voltage for half-maximal activation (in $\mathrm{mV}$ ).

\section{Results}

Trans-splicing ribozyme design. We modified the Tetrabymena group I intron ribozyme to perform a trans-splicing reaction to restore the wild-type cClC-1 sequence. An important sequence requirement for ribozymemediated trans-splicing is the formation of the nonWatson-Crick G:U base pair between the ribozyme and the target RNA (8). Initially, we employed an RNA mapping technique to determine which uridines within the target transcript (T268M mutant mRNA) are ribozyme accessible. Accessible sites within T268M mRNAs were determined by incubating a random-IGS ribozyme library with in vitro transcribed T268M mRNA in a cell-free trans-splicing reaction. Seven accessible uridines were identified by sequencing these reaction products, and the uridine at nucleotide position 712 $\left(U_{712}\right)$ was chosen as our target because it was the $5^{\prime}$ site nearest to T268M (nucleotide 803, Figure 1). A ribozyme targeting $\mathrm{U}_{712}$ with a six-nucleotide IGS and a 3' exon corresponding to nucleotides 713-4,717 of cClC- 1 was constructed, and trans-splicing activity was confirmed in a cell-free system (data not shown).

Previous reports have suggested that a six-nucleotide IGS-containing ribozyme exhibits no activity when expressed from the nucleus in mammalian cells (B.A. Sullenger, unpublished results). Therefore, for use in cells, a ribozyme (designated $\mathrm{RzU}_{712} \mathrm{AS}$ ) directed at $\mathrm{U}_{712}$ was designed with modifications that have been shown to increase activity, including a nine-nucleotide IGS, a P10 helix, and a 33-nucleotide antisense region (Figure 1) (11, 14). Because the antisense region targets RNA sequence identical to analogous sequence in the $3^{\prime}$ exon of the ribozyme, silent mutations were engineered into the $3^{\prime}$ exon to prevent ribozyme inactivation by intramolecular base pairing. This alteration also served as a sequence tag for distinguishing trans-spliced products from nonspliced RNAs in subsequent hybridization-based assays.

In vitro repair of T268M $m R N A$. The ability of the ribozyme to perform trans-splicing was tested in HEK-293 cells stably expressing T268M (T268M-stable cells). This human embryonic kidney cell line does not express endogenous ClC-1 (15). Cells were transfected with plasmid DNA encoding the ribozyme and total RNA was isolated 20 hours later. Allele-specific RT-PCR confirmed trans-splicing in ribozyme-transfected cells, whereas cells transfected with a catalytically inactive ribozyme did not exhibit trans-splicing reaction products (Figure 2a). To demonstrate that the RNA repair reaction occurred in ribozyme-treated cells and not during the RNA isolation procedure, ribozyme-transfected HEK-293 cells were mixed with T268M-stable cells prior to RNA isolation. No trans-splicing product was observed in this experiment (Figure 2a). The intended trans-spliced 

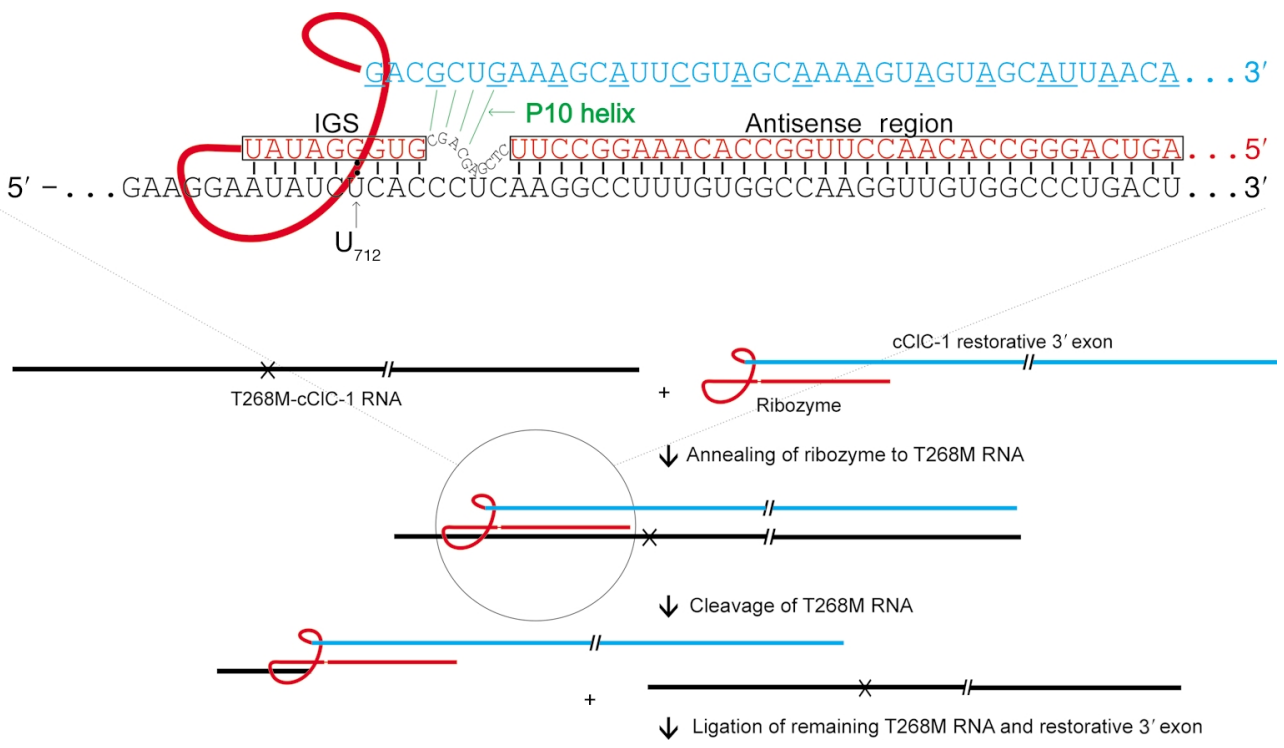

Correctly trans-spliced cCIC-1 RNA

\section{Figure 1}

Scheme for ribozyme-mediated repair of mutant cClC-1 mRNA. The ribozyme (red) contains the IGS, which base pairs to a complementary site on the mRNA target (black) upstream of the T268M mutation $(x)$. The ribozyme catalyzes the cleavage of T268M mRNA and then the ligation of the remaining target sequence and the wild-type $3^{\prime}$ exon sequence (blue), thus restoring the correct translational reading frame of $\mathrm{cClC}-1$. The circled region is expanded to show the IGS, P10 helix, and antisense region interactions. Underlined bases indicate the sequence tag of silent mutations.

junction was confirmed by DNA sequence analysis of RT-PCR products from ribozyme-transfected T268M stable cells (Figure 2b), indicating reaction fidelity.

Efficiency of trans-splicing reactions. The efficiency of mRNA repair was assessed with real-time quantitative RT-PCR using TaqMan chemistry. For these experiments, efficiency was defined as the proportion of total target RNAs that were correctly trans-spliced. Primer sets were designed to specifically amplify trans-spliced and nonspliced products in separate reactions, while a fluorogenic oligonucleotide probe was used to detect either trans-spliced or nonspliced products. The RNA repair efficiency assay was validated using various ratios of mutant and wild-type plasmid DNAs to represent varying levels of repair efficiency. Expected and actual values were correlated linearly $\left(R^{2}=0.999\right)$.

Efficiency of the trans-splicing reaction was determined using total RNA isolated from T268M-stable cells transfected with plasmids encoding either an active or inactive ribozyme. To enable visualization and quantification of transfected cells, we engineered ribozyme constructs in a bicistronic plasmid that drives expression of EGFP and ribozyme from separate promoters. To facilitate construction of these plasmids, the $3^{\prime}$ untranslated region (UTR) of cClC-1 was partially truncated, leaving a 3.2-kb 3' exon. Because transfection occurs with variable efficiency, we corrected the measured level of nonspliced product in each experiment using an approach outlined in Methods. Ribozymetransfected cells exhibited an RNA repair efficiency of $1.2 \% \pm 0.1 \%(n=16)$, while cells transfected with inactive ribozyme exhibited no repair $(n=11)$ (Figure 3). No trans-splicing events were detected in nontransfected T268M-stable cells $(n=4)$ or mix controls $(n=4)$.

Factors potentially affecting trans-splicing efficiency. Several factors may be contributing to the low RNA repair efficiency observed in our study, including insufficient ribozyme excess or inadequate colocalization of ribozyme and target RNAs. Quantitative RT-PCR was used to assess the relative levels of ribozyme and T268M RNA. In our trans-splicing experiments, fold excess of ribozyme was $7.6 \pm 0.3(n=12)$. RNA localization within cellular compartments is sometimes dictated by the presence of cis-acting signals within the $3^{\prime}$ UTR $(16,17)$. However, inclusion of the complete $3^{\prime}$ UTR of cClC- 1 within the $3^{\prime}$ exon did not improve RNA repair efficiency significantly $(1.4 \% \pm 0.1 \%, n=8)$.

Rescue of chloride channel function. Because we are targeting a defective ion channel for repair, we can directly assess restored protein activity in single cells using electrophysiological techniques. The whole-cell configuration of the patch clamp technique was used to record chloride currents in T268M-stable cells following transient transfection of either active or inactive ribozyme-encoding plasmid DNA. Figure 4, a and b, illustrates typical current traces obtained from cells expressing either wild-type cClC-1 or T268M. Wholecell currents carried by wild-type cClC- 1 exhibit fast activation and deactivation at hyperpolarizing potentials, and no current at depolarizing potentials (Figure 4a). However, in T268M-expressing cells, membrane hyperpolarization causes the inward current amplitude to rise 


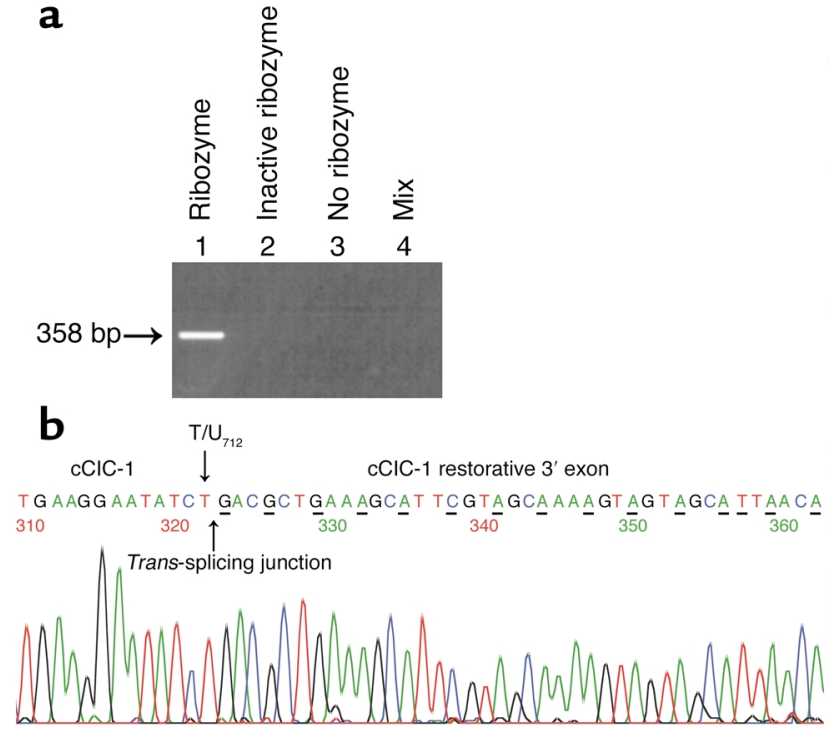

Figure 2

In vitro T268M repair. (a) Allele-specific RT-PCR amplification of a 358-bp trans-spliced product from cells treated with ribozyme. Inactive ribozyme-treated T268M-stable cells, nontransfected T268M-stable cells, and a mix of ribozyme-treated HEK-293 cells and T268M-stable cells failed to yield a product. (b) Representative sequence trace of repaired $c \mathrm{ClC}-1$ sequence. The targeted uridine $\left(T / \mathrm{U}_{712}\right)$ and the transsplice junction are labeled. Underlined bases represent the sequence tag.

instantaneously, followed by a slower deactivation to a steady-state level, while membrane depolarization triggers slowly activating outward currents (Figure 4b). These differences are due to a shift in the voltage dependence of activation to more positive potentials in the mutant, causing fewer channels to be available for sarcolemmal repolarization (13). Correction of this biophysical defect would be expected to abolish the myotonic phenotype associated with this mutation.

We performed similar electrophysiological analyses on cells transfected with plasmids encoding the active or inactive ribozyme and EGFP as a reporter. Only EGFPpositive cells were tested. Figure 4, c-f, illustrates representative "tail" currents from a wild-type cClC-1 cell, a T268M cell, and two ribozyme-transfected cells. In this experiment, tail currents were measured at a test potential of $-120 \mathrm{mV}$ (arrow in Figure 4 inset) and represent the activity of channels opened by the preceding voltage. The bold black line in each family of traces represents the tail current recorded after a 1.4-second prepulse to $0 \mathrm{mV}$. In wild-type cClC-1-expressing cells (Figure 4c), channels are fully activated at this potential, while very little channel activation $(-13 \%)$ occurs in T268M-expressing cells (Figure 4d). In Figure 4, e and f, current traces obtained from two ribozyme-expressing cells illustrate a spectrum of biophysical rescue with either complete (Figure $4 \mathrm{e}$ ) or partial (about $40 \%$ activation, Figure $4 \mathrm{f}$ ) restoration of wild-type chloride channel function.

We evaluated the degree of functional restoration in several individual cells by examining the voltage dependence of activation and determining the voltage at which half-maximal activation $\left(V_{1 / 2}\right)$ occurs (Figure 5 ). We categorized the magnitude of repair as full, partial, or none based upon the observed changes in $V_{1 / 2}$ (Figure 5a). Full repair was defined as $V_{1 / 2}$ falling within $5 \mathrm{mV}$ of wild-type cClC-1, a value well within one SD of the mean. No repair was defined as $V_{1 / 2}$ values falling within two SDs (approximately $18 \mathrm{mV}$ ) of the mean of T268M. The remaining values were considered indicative of partial repair (i.e., a mixed population of mutant and corrected channels). Thirteen of 71 ribozyme-transfected cells (18\%) exhibited a significant shift of $V_{1 / 2}$ to more negative potentials. Two of these cells demonstrated a complete shift in the activation curve to voltages that are not significantly different from wild-type cClC-1, suggesting that the transsplicing ribozyme has the ability to fully rescue the mutant phenotype. Figure $5 \mathrm{~b}$ illustrates the activation $V_{1 / 2}$ for wild-type cClC- 1 (square) and T268M (diamond). The $V_{1 / 2}$ values from 13 ribozyme-transfected cells that exhibited some degree of biophysical correction are shown individually (filled circles), while the mean $V_{1 / 2}$ of nonrepaired cells is shown as a single data point (open circle). T268M-stable cells transfected with the inactive ribozyme exhibit no significant change in activation (triangle). These data indicate that a trans-splicing ribozyme is capable of restoring wild-type function to a mutant skeletal muscle chloride channel, but there is considerable cell-to-cell variability in reaction efficiency.

\section{Discussion}

In this paper, we describe the engineering of a transsplicing ribozyme to correct a mutant skeletal muscle chloride channel mRNA. The ribozyme was used to repair T268M transcripts in a mammalian cell line by trans-splicing a 4-kb restorative 3 ' exon. Sequence analysis indicated that this was a high-fidelity reaction. Efficiency of ribozyme-mediated RNA repair determined in a population of cells was not high (1.2\%). However, using single-cell electrophysiological methods, we

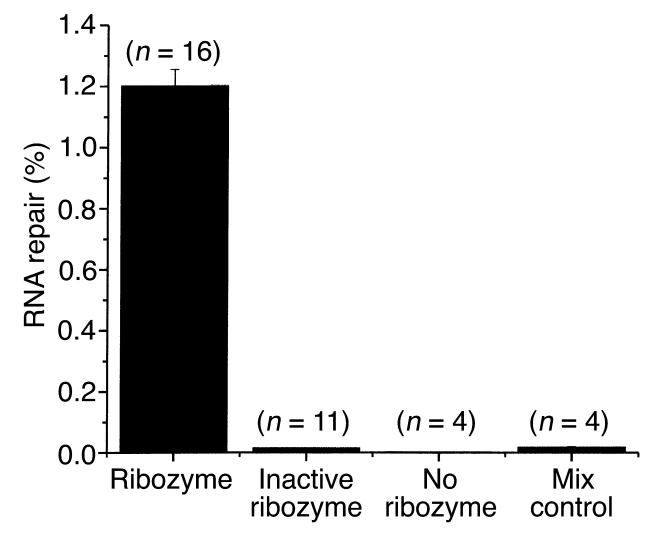

\section{Figure 3}

Trans-splicing reaction efficiency. Real-time quantitative RT-PCR was performed to assess the efficiency of the trans-splicing reaction in a pool of cells. T268M-stable cells were transfected with ribozyme or inactive ribozyme. A mix control was included in which ribozymetreated HEK-293 cells and nontransfected T268M-stable cells were combined prior to RNA isolation. Error bars indicate SEM. 


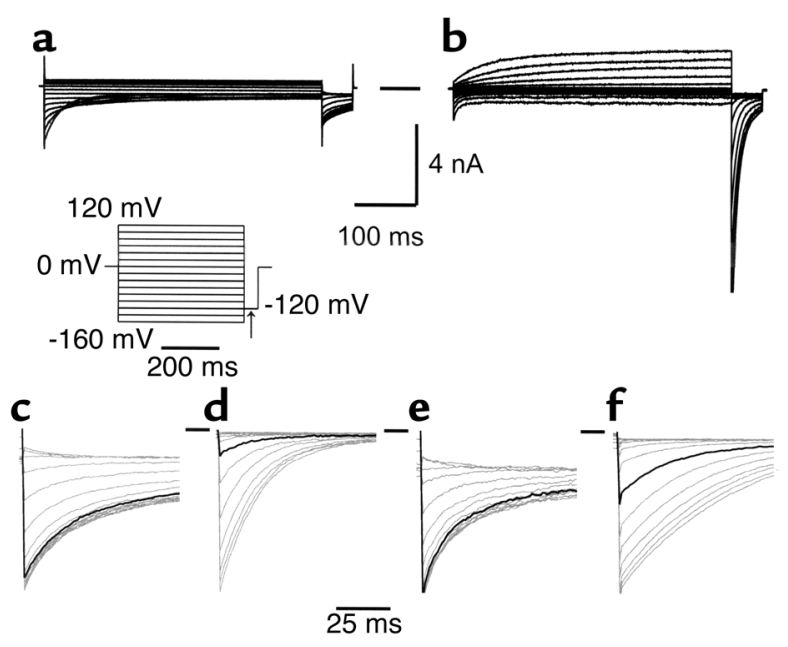

Figure 4

Ribozyme-mediated functional repair of mutant cClC-1. ( $\mathbf{a}$ and $\mathbf{b}$ ) Representative whole-cell voltage-clamp recordings of HEK-293 cells transiently expressing wild-type cClC-1 (a) and stably expressing T268M (b). (c-f) Representative tail current traces from wild-type cClC-1 (c), T268M (d), and two ribozyme-transfected cells: full repair (e) and partial repair (f). Current traces recorded at $-120 \mathrm{mV}$ following a $0-\mathrm{mV}$ prepulse are shown as bold black lines in $\mathbf{c}-\mathbf{f}$.

demonstrated a range of significant functional restoration, with some cells exhibiting complete rescue of the chloride channel defect. Of the individual cells analyzed, $18 \%$ (13/71) exhibited significant biophysical improvement in channel activity, with wild-type functional properties restored in a few cells. These data provide the first direct demonstration of the complete restoration of protein function using trans-splicing ribozymes.

The efficiency of the trans-splicing reaction is a critical issue. Our results suggest that the reaction efficiency measured in a population of cells is low but does not strictly reflect the level of repair in individual cells. We can speculate about the various explanations for this phenomenon. The size of the $3^{\prime}$ exon used by our ribozyme is probably not a factor, as we have observed similar levels of repair efficiency with constructs having smaller exons (C.S. Rogers, unpublished results). Variable ribozyme delivery and expression is the simplest explanation for the wide range of activity observed at

\footnotetext{
Figure 5

Spectrum of ribozyme-mediated restoration of cClC-1 function. (a) Voltage dependence of activation for wild-type cClC-1 (squares), T268M (diamonds), and three representative ribozyme-transfected T268M-stable cells (black lines). Dotted curves represent two SDs from the mean of T268M. (b) Scatter diagram illustrating the $V_{1 / 2}$ determined for cells expressing wild-type cClC-1, T268M, and ribozyme-treated T268M. Data for the 13 cells demonstrating a significant level of functional repair are shown as individual data points (filled circles). Mean values for wild-type cClC-1 (square, $n=12$ ), untreated T268M (diamond, $n=52$ ), ribozyme-treated T268M exhibiting no repair (open circle, $n=58$ ) and inactive ribozyme-treated T268M (triangle, $n=11$ ) are shown as single points. Dotted line represents two SDs from the mean of T268M. Error bars represent SEM. (In some cases, the data symbols are larger than the error bars.)
}

the single-cell level. We monitored transfection efficiency by coexpression of EGFP from the same plasmid and attempted to correct RNA repair efficiency values accordingly. However, cellular requirements for expression of EGFP and a catalytically active ribozyme may be vastly different. Furthermore, achievement of highly efficient trans-splicing may require a large molar excess of ribozyme well beyond the 7.6-fold ribozyme-to$\mathrm{T} 268 \mathrm{M}$ ratio measured in our in vitro system (a stable cell line overexpressing the target RNA).

Another explanation could be variable efficiency of repair at the molecular level due to variable RNA folding and interaction with RNA-binding proteins that alter site accessibility (18). This idea is supported by the demonstrated importance of mapping a target RNA for accessible sites for the successful design of hammerhead and trans-splicing ribozymes $(9,19)$. Additionally, Lan et al. have shown that two trans-splicing ribozymes targeted to different regions of the same target RNA yielded a higher RNA repair efficiency when used together than either ribozyme alone, presumably because of variable accessibility of individual sites on the target RNA (20). Accordingly, the treatment of cells with a pool of multi-IGS ribozymes may be a way to circumvent variable ribozyme activity.

Studies with hammerhead ribozymes have established the benefit of colocalizing ribozyme and target RNA transcripts to ensure efficient cleavage of substrate $(21,22)$. The expression of identical localization signals within the $3^{\prime}$ UTR of each transcript has been demonstrated to colocalize the RNAs and improve reaction efficiency compared with nonlocalized RNAs (23). This strategy has yet to be investigated for the trans-splicing ribozyme. Furthermore, it is not currently known if cClC- 1 mRNA is specifically localized within a cell. We observed no difference in RNA repair efficiency between ribozymes possessing either complete
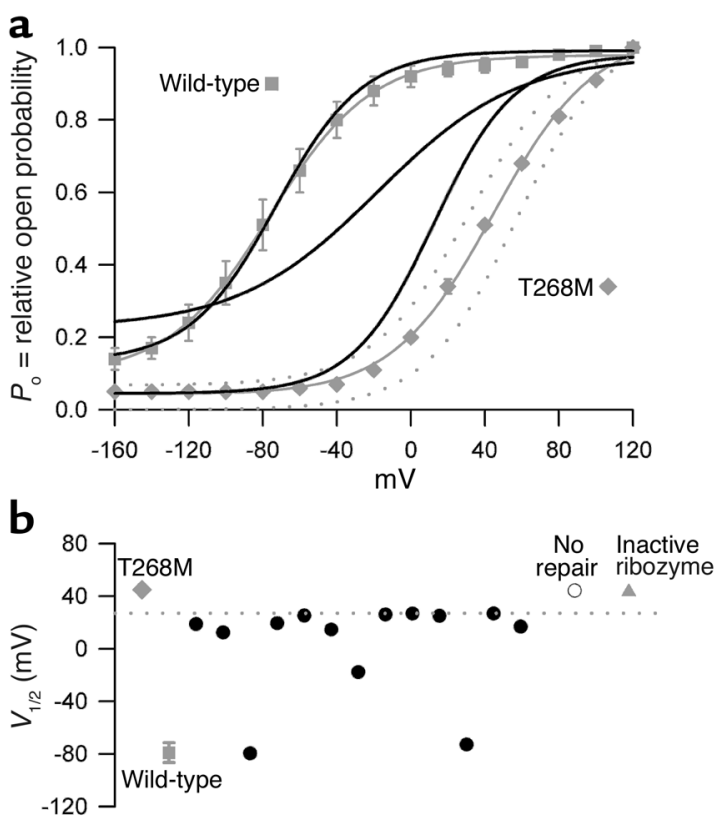
or truncated cClC-1 3' UTRs. Therefore, if the $3^{\prime}$ UTR contains localization signals, its inclusion had no effect on splicing efficiency.

A clearer understanding of cell-to-cell variability in ribozyme activity might be achieved if single-cell RNA repair efficiency could be correlated with single-cell activity data as in our experimental system. We can also speculate that heterogeneity in cell cycle stage may impact trans-splicing efficiency, possibly by affecting the temporal relationship of ribozyme and target mRNA expression $(24,25)$.

Our work also demonstrates that the Tetrahymena group I intron ribozyme can successfully trans-splice a large $(4 \mathrm{~kb})$ exon. Most previously reported RNA repair experiments have demonstrated the trans-splicing of short $3^{\prime}$ exons $(<1 \mathrm{~kb})$ and have not addressed the carrying capacity of these molecules, a potential limitation to the RNA repair strategy. In order to be considered as an effective and widely applicable therapeutic approach, the capacity for trans-splicing larger $3^{\prime}$ exons must be established. The upper limit of $3^{\prime}$ exon length is unknown, but a capacity of at least $4 \mathrm{~kb}$ greatly enhances the potential therapeutic utility of ribozymemediated trans-splicing RNA repair.

An important consideration for any gene therapy strategy is the amount of wild-type expression that must be restored to correct the mutant phenotype. For RNA repair, it is important to consider the percentage of mutant RNA transcripts that must be corrected for clinical benefit. This answer will always be disease-specific and will likely span a wide range. For recessive myotonia congenita, evidence suggests that restoring $20 \%$ of normal sarcolemmal chloride conductance will correct the defect (26). However, the extent of RNA repair required to restore this level of chloride channel function is unknown. A recent study has demonstrated that there is a linear correlation between chloride conductance and $\mathrm{ClC}-1 \mathrm{mRNA}$ levels in rats, indicating a direct relationship between mRNA and chloride channel protein (27). Therefore, it is conceivable that $20 \%$ of defective cClC- 1 transcripts will have to be repaired in order for this strategy to be successful. Although the limitations of our current ribozyme delivery and expression system prevents its immediate therapeutic application, the observation that significant functional correction can be achieved provides the basis for continued optimism.

In summary, we demonstrated functional repair of a mutant chloride channel using a trans-splicing ribozyme. Our work also suggested a striking cell-tocell heterogeneity in RNA repair efficiency that will require further investigation to explain. However, we feel that ribozyme-mediated RNA repair is a viable therapeutic strategy and with additional optimization may be ready for in vivo testing.

\section{Acknowledgments}

The authors are grateful to Megan Olarte for DNA sequencing and computational support, and thank
Laura Bianchi for helpful advice and Niloufar Gillani for technical assistance. We also wish to thank Ron Emeson and Meredith Long for critical reading of this manuscript. This work was supported by NIH grants AR-44506 (to A.L. George, Jr.), DK-61359 (to C.G. Vanoye), and GM-07628 (to C.S. Rogers), and a grant from the Muscular Dystrophy Association (to A.L. George, Jr.).

1. Watanabe, T., and Sullenger, B.A. 2000. RNA repair: a novel approach to gene therapy. Adv. Drug Deliv. Rev. 44:109-118.

2. Puttaraju, M., Jamison, S.F., Mansfield, S.G., Garcia-Blanco, M.A., and Mitchell, L.G. 1999. Spliceosome-mediated RNA trans-splicing as a tool for gene therapy. Nat. Biotechnol. 17:246-252.

3. Sullenger, B.A., and Cech, T.R. 1994. Ribozyme-mediated repair of defective mRNA by targeted, trans-splicing. Nature. 371:619-622.

4. Jones, J.T., Lee, S.W., and Sullenger, B.A. 1996. Tagging ribozyme reaction sites to follow trans-splicing in mammalian cells. Nat. Med. 2:643-648.

5. Jones, J.T., and Sullenger, B.A. 1997. Evaluating and enhancing ribozyme reaction efficiency in mammalian cells. Nat. Biotechnol. 15:902-905.

6. Sullenger, B.A., and Gilboa, E. 2002. Emerging clinical applications of RNA. Nature. 418:252-258.

7. Inoue, T., Sullivan, F.X., and Cech, T.R. 1986. New reactions of the ribosomal RNA precursor of Tetrahymena and the mechanism of self-splicing. J. Mol. Biol. 189:143-165.

8. Been, M.D., and Cech, T.R. 1986. One binding site determines sequence specificity of Tetrahymena pre-rRNA self-splicing, trans-splicing, and RNA enzyme activity. Cell. 47:207-216.

9. Lan, N., Howrey, R.P., Lee, S.W., Smith, C.A., and Sullenger, B.A. 1998. Ribozyme-mediated repair of sickle beta-globin mRNAs in erythrocyte precursors. Science. 280:1593-1596.

10. Phylactou, L.A., Darrah, C., and Wood, M.J. 1998. Ribozyme-mediated trans-splicing of a trinucleotide repeat. Nat. Genet. 18:378-381.

11. Watanabe, T., and Sullenger, B.A. 2000. Induction of wild-type p53 activity in human cancer cells by ribozymes that repair mutant p53 transcripts. Proc. Natl. Acad. Sci. USA. 97:8490-8494.

12. Hamill, O.P., Marty, A., Neher, E., Sakmann, B., and Sigworth, F.J. 1981. Improved patch-clamp techniques for high-resolution current recording from cells and cell-free membrane patches. Pflugers Arch. 391:85-100.

13. Rhodes, T.H., et al. 1999. A missense mutation in canine C1C-1 causes recessive myotonia congenita in the dog. FEBS Lett. 456:54-58.

14. Kohler, U., Ayre, B.G., Goodman, H.M., and Haseloff, J. 1999. Transsplicing ribozymes for targeted gene delivery. J. Mol. Biol. 285:1935-1950.

15. Fahlke, C., Rosenbohm, A., Mitrovic, N., George, A.L., Jr., and Rudel, R. 1996. Mechanism of voltage-dependent gating in skeletal muscle chloride channels. Biophys. J. 71:695-706.

16. Oleynikov, Y., and Singer, R.H. 1998. RNA localization: different zipcodes, same postman? Trends Cell Biol. 8:381-383.

17. Rossi, J.J. 1995. Controlled, targeted, intracellular expression of ribozymes: progress and problems. Trends Biotechnol. 13:301-306.

18. Herschlag, D. 1995. RNA chaperones and the RNA folding problem. J. Biol. Chem. 270:20871-20874.

19. Scherr, M., Reed, M., Huang, C.F., Riggs, A.D., and Rossi, J.J. 2000. Oligonucleotide scanning of native mRNAs in extracts predicts intracellular ribozyme efficiency: ribozyme-mediated reduction of the murine DNA methyltransferase. Mol. Ther. 2:26-38.

20. Lan, N., et al. 2000. Enhancing RNA repair efficiency by combining trans-splicing ribozymes that recognize different accessible sites on a target RNA. Mol. Ther. 2:245-255.

21. Sullenger, B.A., and Cech, T.R. 1993. Tethering ribozymes to a retroviral packaging signal for destruction of viral RNA. Science. 262:1566-1569.

22. Kuwabara, T., et al. 2001. Significantly higher activity of a cytoplasmic hammerhead ribozyme than a corresponding nuclear counterpart: engineered tRNAs with an extended 3 ' end can be exported efficiently and specifically to the cytoplasm in mammalian cells. Nucleic Acids Res. 29:2780-2788.

23. Lee, N.S., Bertrand, E., and Rossi, J. 1999. mRNA localization signals can enhance the intracellular effectiveness of hammerhead ribozymes. RNA. 5:1200-1209.

24. Sanchez, I., and Dynlacht, B.D. 1996. Transcriptional control of the cell cycle. Curr. Opin. Cell Biol. 8:318-324.

25. Brunner, S., et al. 2000. Cell cycle dependence of gene transfer by lipoplex, polyplex and recombinant adenovirus. Gene Ther. 7:401-407.

26. Barchi, R.L. 1975. Myotonia. An evaluation of the chloride hypothesis. Arch. Neurol. 32:175-180.

27. Pierno, S., De Luca, A., Beck, C.L., George, A.L., Jr., and Conte, C.D. 1999. Aging-associated down-regulation of ClC-1 expression in skeletal muscle: phenotypic-independent relation to the decrease of chloride conductance. FEBS Lett. 449:12-16. 